\title{
Sensitivity enhancement of lossy mode resonance- based ethanol sensors by graphene oxide coatings
}

\author{
Miguel Hernaez ${ }^{1}$, Andrew G. Mayes ${ }^{1}$ and Sonia Melendi-Espina ${ }^{2}$ \\ ${ }^{1}$ School of Chemistry, ${ }^{2}$ School of Mathematics \\ University of East Anglia \\ Norwich, UK \\ m.hernaez@uea.ac.uk
}

\begin{abstract}
A layer by layer (LbL) coating made of polyethyleneimine (PEI) and graphene oxide (GO) is deposited onto an optical fiber ethanol sensor based on lossy mode resonances (LMR) in order to improve its response. The new sensor including the PEI/GO coating shows a better linearity and a sensitivity four times higher than the sensor without the coating. To our knowledge, this is the first time that a LbL coating made of PEI and GO is included in an optical fiber sensor based on LMR.
\end{abstract}

Keywords-optical fiber sensor; ethanol; lossy mode resonances; graphene oxide

\section{INTRODUCTION}

Graphene has become a trending topic in different scientific fields since Novoselov and Geim reported its successful isolation in 2004 [1]. Its outstanding properties make it an ideal candidate for several applications. However, the synthesis price and complexity make unfeasible the use of graphene in some fields. In this regard, graphene oxide (GO) and reduced graphene oxide (rGO) can be good substitutes of graphene in many applications as they are a good compromise between the interesting properties of graphene and their easier production, variety of deposition methods and more affordable cost. In particular, $\mathrm{GO}$ and $\mathrm{rGO}$ are attracting particular attention in the last years [2,3] for sensing applications due to their high specific surface area, high electronic mobility and low electrical noise.

Among all other sensing strategies, optical fiber sensors have achieved a high impact in the last decades because they offer several advantages over electronic sensors [4]. Particularly, optical fiber sensors are light, small, resistant to harsh environments and high temperatures, biocompatible, immune to electromagnetic fields and electromagnetically passive. These features make them especially suitable for some specific applications, such as biosensing, health care and sensing in offshore, harsh or flammable environments.

In this regard, resonance-based sensing devices are generating the interest of the scientific community within the last years due to their versatility, high sensitivity and robustness. In particular, lossy mode resonance (LMR) constitute a promising research field that could compete with the largely established surface plasmon resonance-based sensors since they do not depend on the polarization of light and enlarge the range of materials available for sensor surface coating from noble metals to metal oxides, polymers and many others. When an optical waveguide is coated with a thin-film, the propagation of light is affected. If the refractive index of the coating has a nonzero imaginary part, it introduces losses that can produce electromagnetic resonances. Depending on the properties of the waveguide, the coating and the external medium, different cases of electromagnetic resonances can be distinguished, LMR among them. These LMRs produce an absorption band in the transmitted spectrum at a determined wavelength. A shift of this resonance peak can be observed when the optical conditions of the system change, i.e., when there is a variation in the refractive index of the coating or the surrounding medium. Consequently, if a thin-film sensitive to some analyte is deposited onto a waveguide and used as resonance-supporting coating, a variation in the concentration of this analyte will produce a measurable shift of the LMR peak [5].

The aim of this work is to confirm the suitability of GO coatings to improve the performance of LMR-based optical fiber sensors. To reach this goal, an LMR optical fiber ethanol sensor based on a sputtered $\mathrm{SnO}_{2}$ coating [6] has been fabricated and characterized. Then, it has been modified by depositing a multilayer coating including polyethyleneimine (PEI) and GO onto the $\mathrm{SnO}_{2}$ layer following the layer-by-layer (LbL) technique described in [7]. Finally, this device has been also characterized and both responses have been compared in terms of sensitivity, linearity and hysteresis.

To our knowledge, this is the first time that a LbL coating made of PEI and GO is included in an optical fiber sensor based on LMR.

\section{EXPERIMENTAL SECTION}

The experimental aspects of this research, such as the fabrication of the sensing device and the characterization setup and procedure are explained in this section.

\section{A. Sensor fabrication}

The fabrication of the sensor involves several steps. First, a $10 \mathrm{~cm}$ fragment of a $200 \mu \mathrm{m}$-core multimode optical fiber (FT200EMT, purchased from Thorlabs, Inc.) was prepared. Its cladding was chemically removed and the resultant bare core was sonicated and cleaned in both detergent and acetone and rinsed with ultrapure water. After the cleaning process, it was coated during 150 seconds with $\mathrm{SnO}_{2}$ using a sputter coater (K675XD from Quantum Technologies). In order to obtain an even film, the substrate was subjected to continuous rotation 
during the sputtering process. A $2 \mathrm{~cm}$ fragment of the resultant device was perpendicularly cleaved using a NorthLab ProCleave LD II. This device will be called Sensor 1 from this point. It was spliced to $200 \mu \mathrm{m}$ optical fiber core pigtails in both extremes using a Fitel S178A fusion splicer in order to connect it to rest of the characterization setup.

Sensor 2 is the result of depositing a PEI/GO thin film onto Sensor 1 by means of the layer-by-layer method. A $0.5 \mathrm{mg} / \mathrm{ml}$ GO (Graphenea Inc.) solution and $2 \mathrm{mg} / \mathrm{ml}$ PEI (Sigma-Aldrich) solution in DI water were prepared and left stirring overnight. Just before starting the deposition, the GO solution was sonicated during 2 hours to avoid aggregation. At the same time, the substrate was immersed into a $1 \mathrm{M}-\mathrm{KOH}$ solution during 30 minutes and thoroughly rinsed with DI water.

The LbL deposition technique started with the immersion of the substrate into the PEI solution during 5 minutes. After this time, the substrate was rinsed with DI water during 3 minutes in order to remove the excess of material and dried on air during 3 more minutes. Then, the substrate was immersed into the GO solution to form the first bilayer of PEI/GO. The final coating consisted in the repetition of the whole LbL process until the thickness reached the desired value.

\section{B. Device characterization}

The typical optical fiber transmission setup shown in Fig. 1 was used to collect the spectra transmitted through the sensor. A halogen white light source (HL2000, Oceanoptics Inc.) was connected to one of the optical fiber pigtails in order to couple light into the sensing device. The coupled light passes through the sensor and arrives to a spectrometer (Oceanoptics USB2000) connected to the other pigtail. In order to characterize the response of Sensor 1 and Sensor 2 as ethanol sensors the sensitive device was immersed into aqueous ethanol solutions at $\mathrm{v} / \mathrm{v}$ concentrations of $0 \%, 20 \%, 40 \%, 60 \%, 80 \%$ and $100 \%$.

\section{RESULTS}

In this section, the characterization of both devices as ethanol sensors as well as the characterization of the PEI/GO coating deposited to build Sensor 2 are presented.

\section{A. Sensor 1}

Sensor 1 was sequentially immersed in ethanol water solutions with concentrations from 0 to $100 \% \mathrm{v} / \mathrm{v}$ and from $100 \%$ to $0 \% \mathrm{v} / \mathrm{v}$. The transmitted spectra collected using the setup shown in Fig. 1 are shown in the inset of Fig. 2.

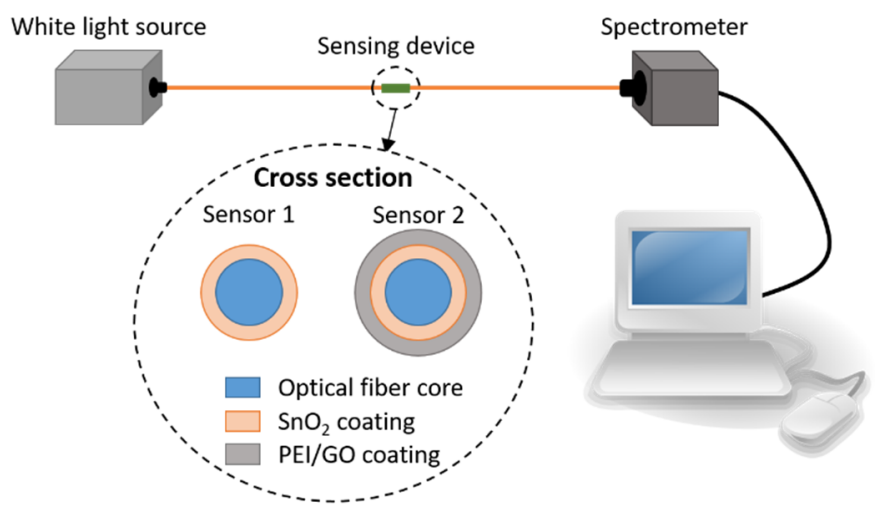

Fig. 1. Optical fiber experimental setup and sensors schematic structure

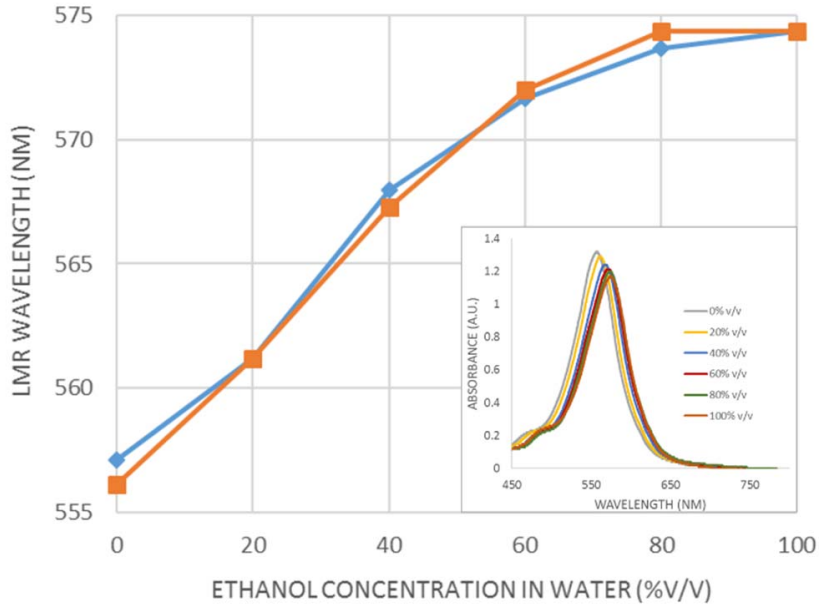

Fig. 2. Shift of the LMR absorption peak generated by Sensor 1 when it is immersed in aqueous ethanol solutions with different concentrations from 0 to $100 \% \mathrm{v} / \mathrm{v}$ (blue line) and from 100 to $0 \% \mathrm{v} / \mathrm{v}$ (orange line). The absorption spectra corresponding to increasing concentrations of ethanol are shown in the inset.

An LMR absorption peak shifting to the red as the ethanol concentration is increasing can be observed. This shift can be more clearly appreciated in the main graph of Fig. 2, where central wavelength of the LMR peak is represented vs the ethanol concentration. The minimum value of the LMR wavelength $(557 \mathrm{~nm})$ is reached when Sensor 1 is introduced in water $(0 \%$ ethanol) while the maximum $(574 \mathrm{~nm})$ is produced when it is immersed in $100 \%$ ethanol. This shift corresponds to an average sensitivity of $0.172 \mathrm{~nm} / \%$ in the whole range of ethanol concentrations. This graph can be approximated by a linear function with $\mathrm{R}^{2}=0.923$. It is important to note that the sensitivity for ethanol concentrations higher than $80 \%$ is almost negligible. The hysteresis value of Sensor 1 is $3.14 \%$, as the differences between both lines (blue for increasing and orange for decreasing ethanol concentrations) are very low.

\section{B. Sensor 2}

After Sensor 1 was characterized, a multilayer coating of $\mathrm{PEI} / \mathrm{GO}$ was deposited onto the $\mathrm{SnO}_{2}$ layer. The deposition process was monitored by capturing the transmission spectra after each bilayer, with the sensor completely dry. Fig. 3 shows the shift of the LMR peak during this process.

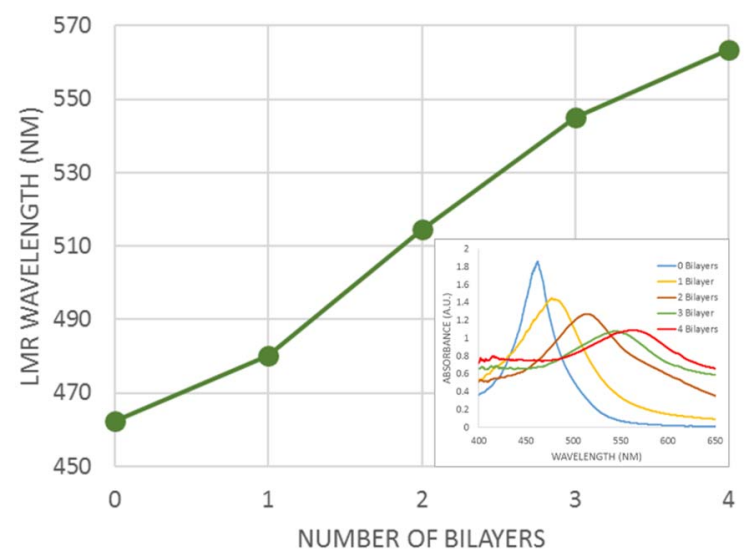

Fig. 3. Shift of the LMR absorption peak generated by the device during the deposition of the PEI/GO coating. 
Before starting the deposition, the LMR was centered in 462 $\mathrm{nm}$. As far as PEI/GO bilayers were added to the coating, the LMR shifted to the red. In Fig. 3 (inset) it can be also appreciated how the shape of the peak was gradually becoming wider and flatter during the deposition. The deposition ended after four bilayers, when the LMR was centered at $563 \mathrm{~nm}$, in order to avoid that it fell beyond the spectrometer operation range during the characterization of the sensor and its shape became too flat to be distinguished. In Fig. 4 SEM (a) and AFM (b) images of the PEI/GO coating are shown. In these pictures, a surface with "wrinkles", typically found in thin-films that include GO nanosheets, can be observed.

The characterization procedure followed with Sensor 1 was exactly replicated with Sensor 2. Fig 5 shows the response of this device to increasing (blue line) and decreasing (orange line) concentrations of ethanol. When Sensor 2 was immersed in water, the LMR was centered in $613 \mathrm{~nm}$. It gradually shifted to the red as far as the ethanol concentration was increasing until it reached its maximum at $680 \mathrm{~nm}$ when the sensor was immersed in pure ethanol. This behavior corresponds to an average sensitivity of $0.676 \mathrm{~nm} / \%$, four times better than the sensitivity of Sensor 1. If this response is approximated by a linear function the value of $R^{2}$ is 0.982 . This means that Sensor 2 response shows a better linearity than Sensor 1, as can be noticed by just looking at the graph. Consequently, the sensitivity is almost constant in the whole range of ethanol concentrations and the device is still sensitive for values higher than $80 \% \mathrm{v} / \mathrm{v}$. Additionally, a low hysteresis of $3.09 \%$ is only appreciable for values of $60 \%$ and $80 \% \mathrm{v} / \mathrm{v}$, being negligible in all other cases.

\section{CONCLUSION}

A significant increase in sensitivity of an optical fiber ethanol sensor based on LMR has been achieved by depositing a multilayer coating made of PEI and GO. Sensor 1, a fragment of multimode uncladded optical fiber coated with a single layer of $\mathrm{SnO}_{2}$ was modified by depositing four bilayers of PEI/GO to obtain Sensor 2. The device was characterized before and after this deposition process by introducing it in aqueous ethanol solutions with concentrations of $0 \%, 20 \%, 40 \%, 60 \%, 80 \%$ and $100 \% \mathrm{v} / \mathrm{v}$. Sensor 1 showed an LMR shift from 557 to $574 \mathrm{~nm}$ while the LMR generated by Sensor 2 shifted from 613 to 680 $\mathrm{nm}$ in the tested range. In other words, the sensitivity of the device became 4 times higher, from $0.172 \mathrm{~nm} / \%$ to $0.676 \mathrm{~nm} / \%$, by just depositing the PEI/GO coating. The linearity of Sensor 2 is also significantly better while the value of the hysteresis were similar for both devices.
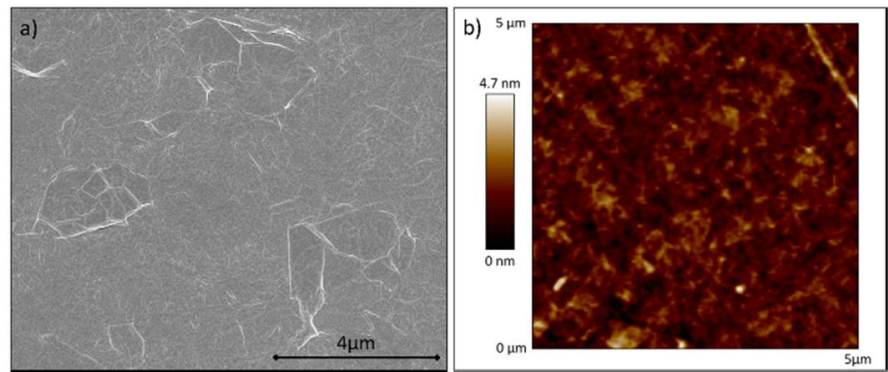

Fig. 4. Images of the superficial structure of the PEI/GO coating: a) SEM and b) AFM.

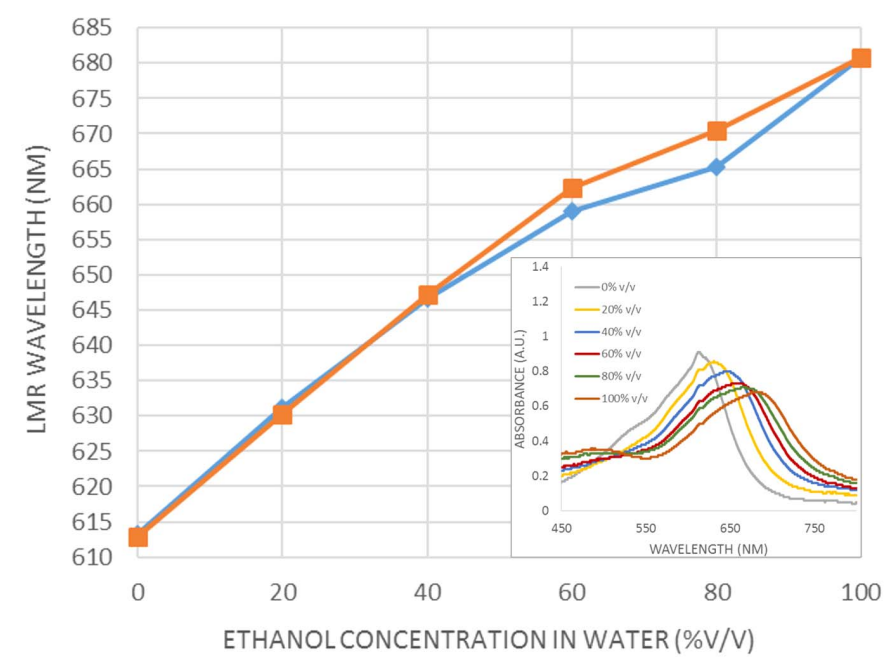

Fig. 5. Shift of the LMR absorption peak generated by Sensor 2 when it is immersed in aqueous ethanol solutions with different concentrations from 0 to $100 \% \mathrm{v} / \mathrm{v}$ (blue line) and from 100 to $0 \% \mathrm{v} / \mathrm{v}$ (orange line). The absorption spectra corresponding to increasing concentrations of ethanol are shown in the inset.

To our knowledge, this is the first time that a LbL coating made of PEI and GO is included in an optical fiber sensor based on LMR.

\section{ACKNOWLEDGMENT}

This work was partially supported by the European Commission H2020-MSCA-IF-2015 703185 Research Grant.

We thank our colleagues from UPNA Sensors Research Group (Universidad Publica de Navarra, Spain) who lent us their facilities and provided help to operate them.

\section{REFERENCES}

[1] K. S. Novoselov et al., "Electric field effect in atomically thin carbon films," Science, vol. 306, pp. 666-669, Oct 2004.

[2] Y. Zhao, X. G. Li, X. Zhou, and Y. N. Zhang, "Review on the graphene based optical fiber chemical and biological sensors," Sensors and Actuators B-Chemical, vol. 231, pp. 324-340, Aug 2016.

[3] M. Hernaez et al., "Optical fibre sensors using graphene-based materials: a review," Sensors, vol. 17, no. 1, p. 155, Jan 2017.

[4] X. D. Wang, and O. S. Wolfbeis, "Fiber-optic chemical sensors and biosensors (2008-2012)," Analytical chemistry, vol. 85(2), pp. 487-508, Nov 2012.

[5] I. Del Villar et al., "Optical sensors based on lossy-mode resonances," Sensors and Actuators, B: Chemical, vol. 240, pp. 174-185, March 2017.

[6] P. Sanchez, C. R. Zamarreño, M. Hernaez, I. R. Matias, and F. J. Arregui, "Optical fiber refractometers based on Lossy Mode Resonances by means of SnO2 sputtered coatings," Sensors and Actuators, B: Chemical, vol. 202, pp. 154-159, Oct. 2014.

[7] Q. Nan, P. Li, and B. Cao, "Fabrication of positively charged nanofiltration membrane via thelayer-by-layer assembly of graphene oxide and polyethylenimine fordesalination," Applied Surface Science, vol. 387, pp. 521-528, June 2016. 